\title{
MAXIMAL SEPARABLE SUBFIELDS OF BOUNDED CODEGREE
}

\author{
JAMES K. DEVENEY AND JOHN N. MORDESON
}

\begin{abstract}
Let $L$ be a function field in $n>0$ variables over a field $K$ of characteristic $p \neq 0$. An intermediate field $S$ is maximal separable if $S$ is separable over $K$ and every subfield of $L$ which properly contains $S$ is inseparable over $K$. This paper examines when $\{[L: S] \mid S$ is maximal separable $\}$ is bounded. The main result states that this set is bounded if and only if there is an integer c such that any intermediate field $L_{1}$ over which $L$ is purely inseparable and $\left[L: L_{1}\right]>p^{c}$ must be separable over $K$. Examples are also given where the above bound is $p^{n+1}$ for any $n \geqslant 1$.
\end{abstract}

Let $L$ be a function field in $n(n>0)$ variables over a field $K$ of characteristic $p \neq 0$. An intermediate field $S$ is maximal separable if $S$ is separable over $K$ and every subfield of $L$ which properly contains $S$ is inseparable over $K$. It is clear that $L$ is purely inseparable and finite dimensional over any maximal separable $S$. This paper is concerned with $\{[L: S] \mid S$ is maximal separable $\}$. Such an $S$ is distinguished if $L \subseteq K^{p^{-x}}(S)$, that is, $L$ is contained in a field obtained from $S$ by adjoining only roots of elements of $K$. Every $L / K$ has distinguished subfields and moreover, $S^{\prime}$ is distinguished if and only if $\left[L: S^{\prime}\right]=\min \{[L: S] \mid S$ is maximal separable [8]. If this minimum is $p^{r}$, then $r$ is called the order of inseparability of $L / K$, denoted inor $(L / K)$. [2] examined the question of when every maximal separable subfield of $L / K$ is distinguished, i.e., $\{[L: S] \mid S$ is maximal separable $\}=$ $\left\{p^{r}\right\}$. Recently Heerema, [7], examined the question of when $\{[L: S] \mid S$ is maximal separable $\}$ is bounded. He showed, for the case where $L$ is of transcendence degree 1 over $K$, that this set is bounded if and only if the algebraic closure of $K$ in $L$ is separable over $K$. This paper continues the investigations begun in [7].

If $\{[L: S] \mid S$ is maximal separable $\}$ is bounded, then any intermediate field $L_{1}$, over which $L$ is not algebraic, must be separable over $K$ (Corollary 6). In some special cases, the converse of this result is also true, and we conjecture it is true in general. The main result, Theorem 10 , gives a characterization of when $\{[L: S] \mid S$ is maximal separable $\}$ is bounded. We also give examples of extensions where $p^{n+1}$ is the bound for $\{[L: S] \mid S$ is maximal separable $\}, n \geqslant 1$.

We will need the following notions. Insep $(L / K)=\log _{p}\left[L: K\left(L^{p}\right)\right]$-the transcendence degree of $L / K$. Since insep $(L / K)=0$ if and only if $L$ is separable over $K, \operatorname{insep}(L / K)$ is a measure of the inseparability of $L / K . \operatorname{Inex}(L / K)=$ $\min \left\{r \mid K\left(L^{p^{r}}\right)\right.$ is separable over $\left.K\right\}$. If $L_{1}$ is an intermediate field of $L / K$, then $\operatorname{inor}(L / K) \geqslant \operatorname{inor}\left(L_{1} / K\right)$, and we have equality if and only if $L^{p^{n}}$ and $K\left(L_{1}^{p^{n}}\right)$ are linearly disjoint over $L_{1}^{p^{n}}$ for all $n\left[1\right.$, p. 656]. If $\operatorname{inor}(L / K)=\operatorname{inor}\left(L_{1} / K\right)$, then $L_{1}$

Received by the editors April 9, 1982 and, in revised form. November 2, 1982.

1980 Mathematics Subject Classification. Primary 12F15, 12F20. 
is called a form of $L / K$. The fields $K\left(L^{(n)}\right)=\left\{x \in L \mid x^{p^{\prime}} \in K\left(L^{p^{\prime \prime \prime}}\right)\right.$ for some $t \geqslant 0\}$ were first introduced in [6]. For $n \geqslant \operatorname{inex}(L / K), K\left(L^{(n)}\right)$ has $K\left(L^{p^{n}}\right)$ as a maximal separable subfield.

Proposition 1. Let $\left\{L_{n} \mid 1 \leqslant n<\infty\right\}$ be a descending chain of intermediate fields of $L / K$. Then $\cap L_{n}$ is separable over $K$ if and only if there exits $n_{0}<\infty$ such that $L_{n_{0}}$ is separable over $K$.

Proof. Inor $\left(L_{1} / K\right) \geqslant \operatorname{inor}\left(L_{2} / K\right) \geqslant \cdots$ by [1, Theorem 1.2, p. 656]. Since this is a nonincreasing sequence of nonnegative integers, it must eventually become constant. Let $n_{0}$ be such that $\operatorname{inor}\left(L_{n_{0}} / K\right)=\operatorname{inor}\left(L_{n_{0}+1} / K\right)=\cdots$. Then $L_{n_{0}+j} / K$ is a form of $L_{n_{0}} / K$ for all $j \geqslant 0$. Hence $\cap L_{n} / K$ is a form of $L_{n_{0}} / K$ by the proof of [1, Theorem 1.4, p. 657]. Thus $\cap L_{n}$ is separable over $K$ if and only if $L_{n_{0}}$ is separable over $K$.

COROllaRy 2. $L / K$ has an infinite descending proper chain of inseparable intermediate fields if and only if there is an intermediate field $L_{1}$ which is inseparable over $K$ and over which $L$ is not algebraic.

Proof. This follows from Proposition 1 and the fact that a finitely generated field extension with an infinite proper chain of intermediate fields cannot be an algebraic field extension.

Let $\bar{K}$ denote the algebraic closure of $K$ in $L$. The following result is [6, Corollary 6, p. 289], however Proposition 1 gives a simple proof.

Corollary 3. $\bar{K} / K$ is separable if and only if $K\left(L^{(n)}\right)=K\left(L^{p^{n}}\right)$ for some $n$.

Proof. $K\left(L^{(n)}\right) \supseteq K\left(L^{p^{n}}\right)$ and has $K\left(L^{p^{n}}\right)$ as a maximal separable extension of $K$ in $K\left(L^{(n)}\right)$. Thus $K\left(L^{(n)}\right)=K\left(L^{p^{n}}\right)$ if and only if $K\left(L^{(n)}\right)$ is separable over $K$. Since $\cap K\left(L^{(n)}\right)=\bar{K}[6$, Theorem 5, p. 289], the result follows from Proposition 1.

Recall that a separable extension of $S$ of $K$ is maximal separable extension of $K$ in $L$ if and only if $L$ is purely inseparable over $S$ and $L^{p} \cap S \subseteq K\left(S^{p}\right)$ [5, Lemma 1.2, p. 46]. In particular, if a relative $p$-basis for $S$ over $K$ remains $p$-independent in $L$, then clearly $L^{p} \cap S \subseteq K\left(S^{p}\right)$. If $L=L_{1}(x)$ where $x$ is transcendental over $L_{1}$, then $L$ is said to be ruled over $L_{1}$.

THEOREM 4. If $L$ is ruled over an intermediate field $L_{1}$ and $L_{1}$ is inseparable over $K$, then $L$ has maximal separable subfields of arbitrarily large codegree.

Proof. Let $L=L_{1}(t)$ and let $\left\{z_{1}, \ldots, z_{r}, w_{1}, \ldots, w_{s}\right\}$ be a relative $p$-basis of $L_{1} / K$ where $\left\{z_{1}, \ldots, z_{r}\right\}$ is a separating transcendence basis of a distinguished subfield $D_{1}$ of $L_{1} / K$ Let $S=D_{1}\left(w_{1}+t^{p^{n}}\right)$. Since $t$ is transcendental over $L_{1}, w_{1}+t^{p^{n}}$ is transcendental over $D_{1}$ and hence $S$ is separable over $K$. Since $w_{1}$ is purely inseparable over $D_{1}, t$, and hence $L$, is purely inseparable over $S$. Since $\left\{z_{1}, \ldots, z_{r}, w_{1}\right.$ $\left.+t^{p^{n}}\right\}$ is a relative $p$-basis of $S$ over $K$ which remains $p$-independent in $L, S$ is a maximal separable extension of $K$ in $L$ by the comments preceeding Theorem 4 . Finally, since $S\left(L_{1}\right)=L_{1}\left(t^{p^{n}}\right), p^{n}=\left[L: S\left(L_{1}\right)\right] \geqslant[L: S]$, and hence we can find maximal separable subfields of arbitrarily high codegree. 
ReFereE's Lemma 5 [7, Footnote, p. 354]. If $L / L_{1}$ is finite dimensional and $L_{1} / K$ has maximal separable subfields of arbitrarily high codegree, then so does $L$.

Proof. Let $S_{1}$ be a maximal separable subfield of $L_{1}$ of high codegree. Then $L_{1} / S_{1}$ is purely inseparable and $L_{1}$ has at most $\log _{p}\left[L_{1}: K\left(L_{1}^{p}\right)\right]$ generators over $S_{1}$. But $\log _{p}\left[L_{1}: K\left(L_{\uparrow}^{p}\right)\right] \leqslant \log _{p}\left[L: K\left(L^{p}\right)\right]=$ some fixed constant [8, Lemma 1, p. 111]. There must be an element, say $b$, of large exponent, say $n$, over $S_{1}$. Let $S$ be a maximal separable extension of $K$ in $L$ containing $S_{1} . S$ exists by Zorn's Lemma. Then $S \cap L_{1}=S_{1}$. Thus $b$ is of exponent $n$ over $S$, and hence $[L: S] \geqslant p^{n}$. Thus $L$ has maximal separable subfields of large codegree.

Corollary 6. If $\{[L: S] \mid S$ is a maximal separable extension of $K$ in $L\}$ is bounded, then any intermediate field $L_{1}$ over which $L$ is not algebraic must be separable over $K$.

Proof. Apply Theorem 4 and Lemma 5.

An intermediate field $L_{1}$ of $L / K$ has the same inseparability over $K$ as does $L$ if and only if $L^{p}$ and $K\left(L_{1}^{p}\right)$ are linearly disjoint over $L_{1}^{p}[8$, Lemma 1, p. 111]. The proof of $[1$, Theorem 1.4, p. 657] shows there is a unique minimal intermediate field $L_{l}$ which has $\operatorname{insep}\left(L_{I} / K\right)=\operatorname{insep}(L / K)$. ( $L_{I}$ is merely the intersection of all subfields of $L_{1}$ with insep $\left(L_{1} / K\right)=\operatorname{insep}(L / K)$.)

THEOREM 7. Assume insep $(L / K)=1$. The following are equivalent.

(1) $\{[L: S] \mid S$ is maximal separable extension of $K$ in $L\}$ is bounded.

(2) Any intermediate field $L_{1}$ over which $L$ is not algebraic must be separable over $K$.

(3) $L$ is algebraic over $L_{l}$.

Proof. (1) implies (2) is Corollary 6. Assume (2). Since $L_{I}$ is inseparable over $K, L$ must be algebraic over $L_{I}$. Assume (3). Let $S$ be maximal separable and let $b \in L \backslash S$ with $b^{p} \in S$. Then $S(b)$ is inseparable over $K$, and hence $S(b) \supseteq L_{I}$. Thus $[L: S] \leqslant$ $p \cdot\left[L: L_{I}\right]$.

EXAMPLE 8. We give a family of extensions $L_{n} / K$ where inor $\left(L_{n} / K\right)=1$ and the bound of Theorem 7 is exactly $p^{n+1}$. Let $L_{n}=K\left(x, z, u z^{p^{n}}+x v+w\right), K=$ $P\left(u^{p}, v^{p}, w^{p}\right)$ where $P$ is a perfect field of characteristic $p \neq 0$ and $\{x, z, u, v, w\}$ is algebraically independent over $P$. $L_{n}$ has a subfield $L_{n_{1}}=K\left(x, z^{p^{n}}, u z^{p^{n}}+x v+w\right)$ which is separable algebraic over its irreducible form [2, Example 11, p. 190] and [2, Corollary 7, p. 188], call it $L_{I}$. Since $\operatorname{inor}\left(L_{n} / K\right)=\operatorname{inor}\left(L_{n_{1}} / K\right)=1, L_{I}$ is the irreducible form of $L_{n} / K$. Clearly $\left[L_{n}: L_{n_{1}}\right]=p^{n}$. Let $S$ be a maximal separable extension of $K$ in $L_{n}$, and let $b \in L_{n} \backslash S$ with $b^{p} \in S$. Then $\operatorname{inor}(S(b) / K)=1$, and hence $S(b)$ must contain $L_{I}$. But $L_{n_{1}}$ is separable algebraic over $L_{I}$, and hence is contained in $S(b)$ since $L_{n}$ is purely inseparable over $S(b)$. Thus $\left[L_{n}: S(b)\right] \leqslant$ $\left[L_{n}: L_{n_{1}}\right]=p^{n}$. Thus $\left[L_{n}: S\right] \leqslant p^{n+1}$. But $K\left(x, u z^{p^{n}}+x v+w\right)$ is a maximal separable extension of $K$ in $L_{n}$ (see the comments preceding Theorem 4) which is of codegree $p^{n+1}$. Thus the bound of Theorem 7 is precisely $p^{n+1}$. 
TheOREM 9. Assume tr.d. $(L / K)=1$. The following are equivalent.

(1) $\bar{K} / K$ is separable.

(2) There is an integer $c$ such that any intermediate field $L_{1}$ over which $L$ is purely inseparable and $\left[L: L_{1}\right]>p^{c}$ must be separable over $K$.

(3) $\{[L: S] \mid S$ is maximal separable $\}$ is bounded.

Proof. Assume (1). The proof is by induction on inor $(L / K)$. The result is trivially true for inor $(L / K)=0$. Assume the result for inor $(L / K) \leqslant n-1$ and let $\operatorname{inor}(L / K)=n$. Let $L$ be purely inseparable over $L_{1}$ and suppose $L_{1}$ is inseparable over $K$. We need to show [ $L: L_{1}$ ] must be bounded for all such $L_{1}$. If $L_{1}$ contains a relatively $p$-independent element $x$ of $L / K$, then $L_{1}$ contains the separable algebraic closure of $K(x)$, denoted $(K(x)) \hat{)}$, in $L$, since $L / L_{1}$ is purely inseparable. By the comments preceeding Theorem $4,(K(x))$ is a maximal separable extension of $K$ in $L$. By [7, Theorem 1, p. 353], the degrees of $L$ over its maximal separable intermediate fields is bounded, and since $L_{1} \supseteq\left(K(x) \hat{)}\right.$, the degree of $L$ over $L_{1}$ is bounded. If $L_{1}$ does not contain a relatively $p$-independent element, then $L_{1} \subseteq$ $K\left(L^{p}\right)$. By [1, Lemma 1.1, p. 656], inor $\left.K\left(L^{p}\right) / K\right)<\operatorname{inor}(L / K)$ when inor $(L / K)$ $>0$. Thus by induction, the degree of $K\left(L^{p}\right)$ over $L_{1}$ is bounded, and hence also the degree of $L$ over $L_{1}$. Clearly (2) implies (1) since $\left[L: \bar{K}\left(L^{p^{\prime \prime 1}}\right)\right]>p^{c}$. [7, Theorem 1, p. 353] shows (1) is equivalent to (3).

THEOREM 10. $\{[L: S] \mid S$ is a maximal separable extension of $K$ in $L\}$ is bounded if and only if there is an integer $c$ such that any intermediate field $L_{1}$ over which $L$ is purely inseparable and $\left[L: L_{1}\right]>p^{c}$ must be separable over $K$.

Proof. If $S$ is maximal separable and $b \in L \backslash S$ with $b^{p} \in S$, then $S(b)$ is inseparable over $K$. Thus, the existence of $c$ guarantees $[L: S(b)] \leqslant p^{c}$ and hence $[L: S] \leqslant p^{c+1}$. Now assume there is a bound on the codegrees of maximal separable subfields. We prove there is a $c$ by induction on the transcendence degree of $L / K$. The case of transcendence degree 1 is Theorem 9. We assume there is a sequence $\left\{L_{n}\right\}$ of subfields of increasing codegree which are inseparable over $K$, with $L / L_{n}$ purely inseparable, and get a contradiction.

Let $x$ be a relatively $p$-independent element of $L / K$. Since the codegrees of maximal separable subfields is bounded, $\bar{K}$ is separable over $K$ [7, Corollary 2, p. 354]. Thus $x$ is transcendental over $K$. Since $x$ is also relatively $p$-independent in $L / K$, any maximal separable extension of $K(x)$ in $L$ is also a maximal separable extension of $K$ in $L$. Thus there is a bound on the codegrees of maximal separable extensions of $K(x)$ in $L$, and hence by induction, there is a $c$ for $L / K(x)$. Since $x$ is transcendental over $K$, each $L_{n}(x)$ is inseparable over $K(x)$. Thus the set of codegrees of the $L_{n}(x)$ is bounded.

Let $\left[L: L_{n}\right]=p^{d_{n}}$ where $d_{n}$ is an increasing sequence. Let $\left[L: L_{n}(x)\right] \leqslant p^{c_{1}}$ where $c_{1}$ is a constant. Consider the finite sequence $a_{1}, a_{2}, \ldots, a_{d}$ defined by $p^{a_{r}}=\left[L_{n}^{p^{-r}} \cap\right.$ $\left.L: L_{n}^{p^{r+1}} \cap L\right]$. Note that $\left[L: L_{n}\right]=p^{a_{1}+a_{2}+\cdots+a_{d_{n}}}=p^{d_{n}}$. Since $\left[L: L_{n}(x)\right] \leqslant p^{c_{1}}$ while $\left[L: L_{n}\right]=p^{d_{n}}, x$ is of exponent at least $d_{n}-c_{1}$ over $L_{n}$. Thus, for $i=1, \ldots, d_{n}$ $-c_{1}, a_{i} \geqslant 1$, and at most the last $c_{1}$ of the $a_{i}$ 's are 0 . Since $a_{1}+\cdots+a_{d_{n}}=d_{n}$, at most $c_{1}$ of the $a_{i}$ 's can exceed 1 . So, we have a finite sequence of increasing length 
$\left(d_{n}\right)$ with at most a fixed number of elements $\left(2 c_{1}\right)$ different from 1 . Thus we can find strings of consecutive l's of increasing length, say $w_{n}$, which begin at least as sequence element $a_{d_{n}-w_{n}}$ for the sequence associated to $L_{n}$. Thus for $s=\operatorname{inor}(L / K)$ +1 , we can find, for large $n$, fields $L_{n}^{\prime} \supseteq L_{n}$ such that $L_{n}^{\prime p^{-1}} \cap L$ is simple over $L_{n}^{\prime}$. Rename this sequence as $\left\{L_{n}\right\}$.

We now want to see that $L_{n}^{p^{-s}} \cap L$ has the same order of inseparability over $K$ as $L_{n}^{p^{-s+1}} \cap L$ has over $K$, that is $L_{n}^{p}{ }^{s+1} \cap L / K$ is a form of $L_{n}^{p^{-s}} \cap L / K$. We can write $L_{n}^{p^{-s}} \cap L=L_{n}(\theta)$ and $L_{n}^{p^{-s+1}} \cap L=L_{n}\left(\theta^{p}\right)$. Now, the increase in the order of inseparability of $L_{n}(\theta)$ depends upon $\min \left\{\max \left\{r \mid \theta^{p^{s}} \in K\left(L_{n}^{(r)}\right)\right\}, s\right\}$ [4, Theorem 2, p. 374]. But this minimum must be $\max \left\{r \mid \theta^{p^{*}} \in K\left(L_{n}^{(r)}\right)\right\}<s$ since $s>\operatorname{inor}(L / K)$ $\geqslant \operatorname{inor}\left(L_{n}(\theta) / K\right)$. Since the increase in the order of inseparability of $L_{n}\left(\theta^{p}\right)=$ $\min \left\{\max \left\{r \mid \theta^{p^{s}} \in K\left(L_{n}^{(r)}\right)\right\}, s-1\right\}$, the increases will be the same, i.e., $L_{n}^{p^{-s+1}} \cap$ $L / K$ is a form of $L_{n}^{p^{-s}} \cap L / K$.

By [3, Theorem 3.3], $L_{n}^{p^{-x+1}} \cap L / K$ has a distinguished subfield $D_{n}$ not contained in any of $L_{n}^{p^{-s}} \cap L$. We claim $D_{n}$ is a maximal separable subfield of $L / K$. Clearly $D_{n} / K$ is separable and $L / D_{n}$ is purely inseparable. Suppose $x^{p} \in D_{n}, x \notin D_{n}$. If $x \in L_{n}^{p^{-s+1}} \cap L$ then $x^{p} \in K\left(D_{n}^{p}\right)$ since $D_{n}$ is maximal separable in $L_{n}^{p^{-s+1}} \cap L$. If $x \notin L_{n}^{p^{-s+1}} \cap L$, then $x$ must be in $L_{n}^{p^{-s}} \cap L$ since $L_{n}^{p^{-s}} \cap L$ is simple over $L_{n}$ by construction. If $x^{p}$ were not in $K\left(D_{n}^{p}\right)$, then by a degree argument $D_{n}(x)$ would be distinguished for $L_{n}^{p^{-s}} \cap L / K$, a contradiction. Thus the sequence of $\left\{D_{n}\right\}$ is a set of maximal separable extensions of $K$ of unbounded codegree, a contradiction. Thus there is a $c$ as in the statement of the theorem.

It is clear that the existence of a $c$ as in the previous theorem implies that any subfield $L_{1}$ over which $L$ is not algebraic must be separable over $K$. The converse is true in the transcendence degree $(L / K)=1$ case, Theorem 9, or the insep $(L / K)=1$ case, Theorem 7 . We conjecture that it is true in general.

\section{REFERENCES}

1. J. Deveney and J. Mordeson, The order of inseparability of fields, Canad. J. Math. 31 (1979), 655-662.

2. D_ Distinguished subfields, Trans. Amer. Math. Soc. 260 (1980), 185-193.

3. D__ Distinguished subfields of intermediate fields, Canad. J. Math. 33 (1981), 1085-1096.

4. Calculation invariants of inseparable field extensions, Proc. Amer. Math. Soc. 81 (1981), $373-376$.

5. __ Splitting and modularly perfect fields, Pacific J. Math. 83 (1979), 45-54.

6. N. Heerema, pth powers of distinguished subfields, Proc. Amer. Math. Soc. 55 (1976), 287-292.

7. _ Maximal separable intermediate fields of large codegree, Proc. Amer. Math. Soc. 82 (1981), $351-354$.

8. H. Kraft, Inseparable Korperenweiterungen, Comment. Math. Helv. 45 (1970), 110-118.

Department of Mathematical Sciences, Virginia Commonwealth University, Richmond, VIRGINIA 23284

Department of Mathematics, Creighton University, Omaha, Nebraska 68178 